\title{
Melting of graphene clusters
}

\author{
Sandeep Kumar Singh, ${ }^{1, *}$ M. Neek-Amal, ${ }^{1,2, \dagger}$ and F. M. Peeters ${ }^{1, \ddagger}$ \\ ${ }^{1}$ Department of Physics, University of Antwerpen, Groenenborgerlaan 171, B-2020 Antwerpen, Belgium \\ ${ }^{2}$ Department of Physics, Shahid Rajaee Teacher Training University, Lavizan, Tehran 16785-136, Iran
}

(Received 10 September 2012; published 11 April 2013)

\begin{abstract}
Density-functional tight-binding and classical molecular dynamics simulations are used to investigate the structural deformations and melting of planar carbon nanoclusters $\mathrm{C}_{N}$ with $N=2-55$. The minimum-energy configurations for different clusters are used as starting configurations for the study of the temperature effects on the bond breaking and rotation in carbon lines $(N<6)$, carbon rings $(5<N<19)$, and graphene nanoflakes. The larger the rings (graphene nanoflakes) the higher the transition temperature (melting point) with ring-toline (perfect-to-defective) transition structures. The melting point was obtained by using the bond energy, the Lindemann criteria, and the specific heat. We found that hydrogen-passivated graphene nanoflakes $\left(\mathrm{C}_{N} \mathrm{H}_{M}\right)$ have a larger melting temperature with a much smaller dependence on size. The edges in the graphene nanoflakes exhibit several different metastable configurations (isomers) during heating before melting occurs.
\end{abstract}

DOI: 10.1103/PhysRevB.87.134103

PACS number(s): 64.70.Nd

\section{INTRODUCTION}

The study of the melting of crystals is one of the important subjects in the field of phase transitions. The melting phenomenon occurs at the surface of bulk materials ${ }^{1}$ and needs a microscopic theory for a deep understanding. Nanoscale molecular clusters due to their size-dependent properties show melting processes different from those of bulk materials and infinite-size two-dimensional materials. The melting of nanoclusters has received considerable attention recently, and it was found that nanoclusters melt typically below their corresponding bulk melting temperature. ${ }^{2-4}$ This is due to the higher chemical reactivity of nanoclusters, which is the consequence of the increased accessible surface area and the presence of more free dangling bonds.

The microscopic behavior of nanoclusters at finite temperature can be understood theoretically using a variety of molecular dynamics (MD) methods ${ }^{5-10}$ and can be determined directly by experiment. ${ }^{11-13}$ In most of the simulations the microscopic structure is characterized in terms of bond lengths and their average fluctuations over many cycles of the MD simulation. ${ }^{14-16}$

Since the discovery of two-dimensional (2D) materials, i.e., graphene ${ }^{17,18}$ and hexagonal boron nitride sheets, ${ }^{19}$ the melting of these new materials has attracted much research. ${ }^{20}$ The new $2 \mathrm{D}$ crystalline materials respond to an increasing temperature by losing their lattice symmetry. For example, Zakharchenko et al. ${ }^{20}$ studied the high-temperature behavior of graphene using atomistic simulations. The melting temperature of graphene was estimated to be about $4900 \mathrm{~K}$. Before melting, first Stone-Wales defects appear because they have the smallest energy barrier. When the temperature is increased further, further eventually spaghettilike carbon chains are formed that spread in 3D. A similar melting process can be found for carbon nanotubes using a much smaller critical Lindemann parameter. ${ }^{21}$ The melting temperature of perfect single-wall carbon nanotubes was estimated to be around $4800 \mathrm{~K} .{ }^{21} \mathrm{In}$ graphene nanoribbons the different types of edges (i.e., zigzag and armchair) affect the melting process differently, e.g., Lee et al. $^{22}$ found that at $2800 \mathrm{~K}$ edge reconstruction occurs in a zigzag ribbon.
In our previous work we found that the minimum-energy configuration for flat carbon clusters of up to $N=5$ atoms consists of a line of carbons atoms ${ }^{23}$ (a linear chain) which is in agreement with results of $a b$ initio $^{24}$ calculations. Carbon planar rings were found for $5<N<19$ and graphene nanoflakes are minimum-energy configurations for larger $N .^{25}$ Here we investigate the effect of temperature on those minimum-energy configurations and find the melting temperature of such small flat carbon clusters as a function of the size of the clusters.

A systematic study of the size dependence of the melting temperature is still lacking, as also of the effect of $\mathrm{H}$ passivation of the edge atoms on the melting process. We present such a study and identify the different fundamental steps in the melting process. We found that graphene nanoflakes have a lower transition temperature as compared to bulk graphene and graphene nanoribbons. We also found that H-passivated clusters exhibit higher melting temperatures than non-H-passivated clusters. In all cases, once clusters have defects they can be in different metastable structures (nonplanar isomers). We compare our results with those found for graphene and graphene nanoribbons. The Lindemann index increases with respect to temperature in all cases while its slope versus temperature increases (decreases) linearly for the ring structures (graphene nanoflakes). Furthermore, using ab initio molecular dynamics simulation we analyze the energy change due to defect formation.

This paper is organized as follows. In Sec. II, we introduce the atomistic model and the simulation method. Section III contains our main results and a discussion of the melting of graphenelike clusters and of H-passivated clusters. Section IV gives information on the topology of the defects. The effect of defects on the total energy is introduced in Sec. V. In Sec. VI, we conclude the paper.

\section{SIMULATION METHOD AND MODEL}

\section{A. Minimum-energy configurations}

The second generation of the Brenner reactive empirical bond-order (REBO) potential ${ }^{26}$ function between carbon atoms is used in the present work. All the parameters for the 
(a)

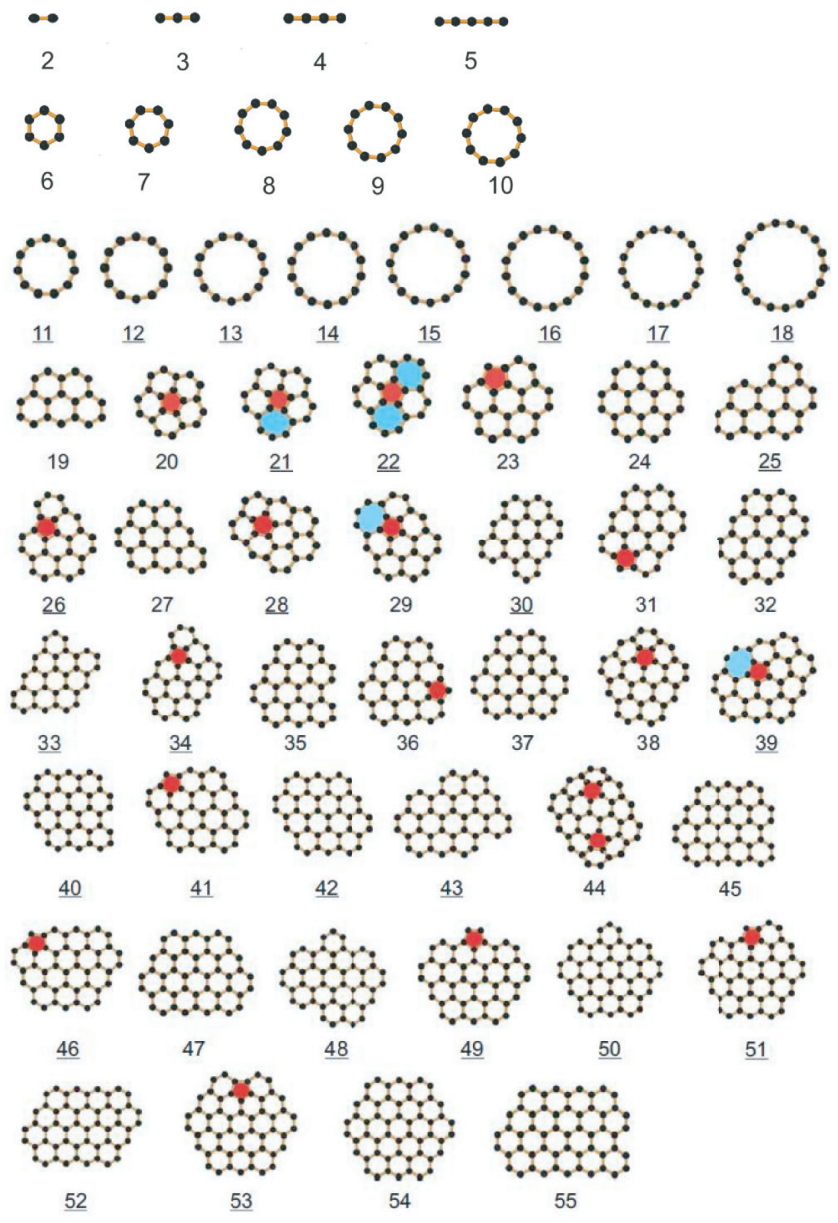

(d)

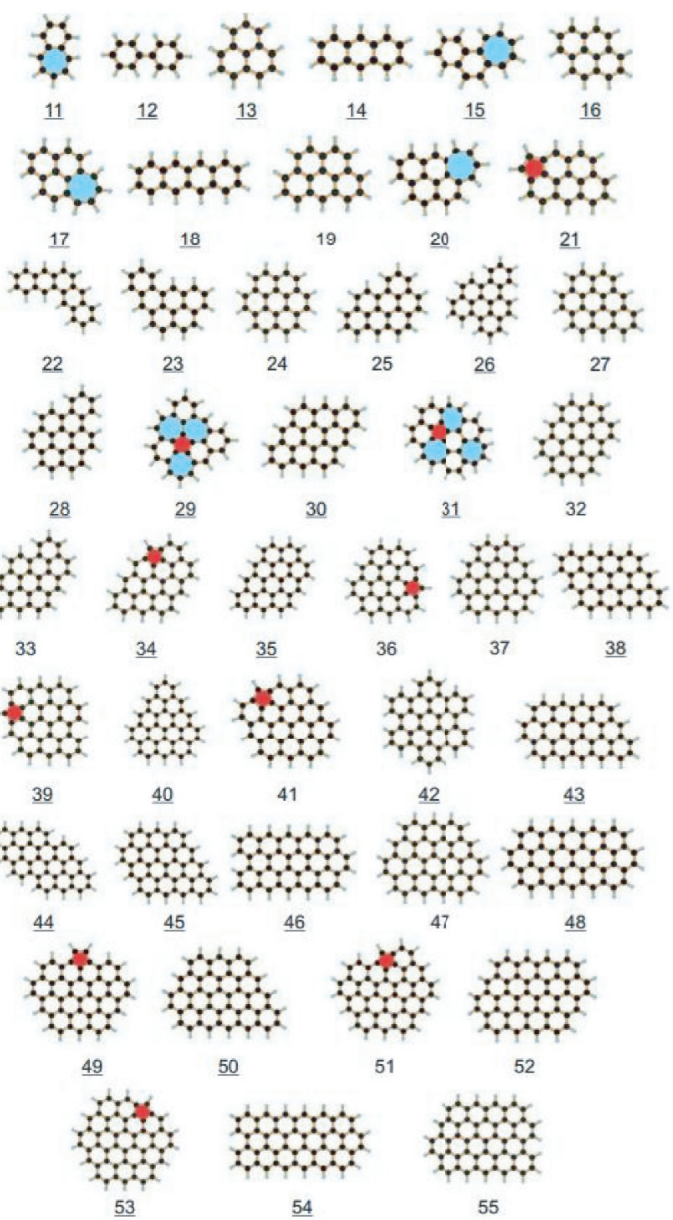

FIG. 1. (Color online) The investigated configurations for carbon lines (a) carbon rings (b), and graphene nanoflakes (c). Pentagons (heptagons) are colored red (blue). By H-passivating the structures in (c) most of them transition to new structures [shown in (d)] which are indicated by underlined numbers.

Brenner potential can be found in Ref. 26 and are therefore not listed here.

In Fig. 1 we depict the minimum-energy configurations for carbon clusters which are carbon lines for up to 5 atoms [Fig. 1(a)], carbon rings for up to 18 atoms [Fig. 1(b)], graphene nanoflakes of up to 55 atoms [Fig. 1(c)] and hydrogen-passivated graphene nanoflakes [Fig. 1(d)]. These configurations were obtained using the conjugate gradient minimization method in our previous works. ${ }^{23,25}$ The carbon line structures are energetically favorable structures among the other possible geometries (isomers) which are shown in Fig. 1 of Ref. 23, i.e., two for $\mathrm{C}_{3}$, six for $\mathrm{C}_{4}$, and 11 for $\mathrm{C}_{5}$. Among all possible carbon nanoclusters (isomers) for $5<N<19$ atoms the ground state is a single ring; see Fig. 1(b). With increase in the number of carbon atoms, graphene nanoflakes are formed which can have pentagon and heptagon defects in addition to common hexagons; see Fig. 1(c). Notice that by passivating the dangling bonds with hydrogen atoms in graphene flakes some structural deformations are possible. In Fig. 1(d) the minimum-energy configurations for hydrogen-passivated graphene nanoflakes [which were obtained by passivating the structures in Fig. 1(c)] are shown. It is interesting to note that most of these minimum-energy-configuration structures have zigzag edges which is due to the higher stability of this kind of edge as compared to armchair edges. ${ }^{27}$

In the present work we study the temperature effects on the structural transition and melting properties of these minimum-energy configurations. Using molecular dynamics simulations, we obtain the new configuration of the abovementioned clusters at a given temperature $T$. This temperature is maintained during the whole simulation by a Langevin thermostat. $^{28}$ The MD time step was taken to be 0.5 fs. Different properties of the clusters were measured during the MD simulation of $10^{6} \mathrm{MD}$ steps (500 ps) at fixed temperature.

\section{B. Density-functional tight-binding molecular dynamics}

In order to have an independent test of the results obtained from the bond-order potential for the melting of graphene nanoflakes, we also performed independent calculations using the density-functional-based tight-binding molecular dynamics (DFTB-MD)approach which is a quantum chemical molecular dynamics (QM-MD) technique based on a tight-binding method using an approximate density-functional formalism. ${ }^{29-31}$ The DFTB method passed several benchmark tests with first-principles density-functional theory ${ }^{29,32,33}$ for 


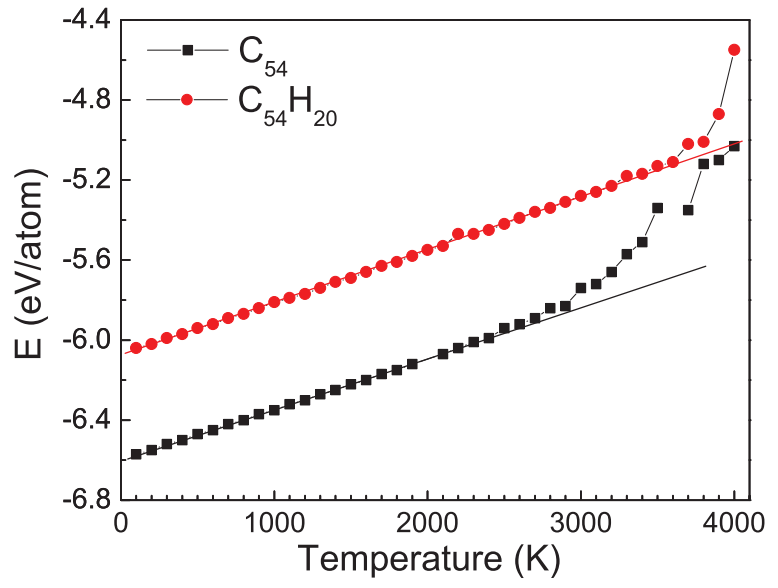

FIG. 2. (Color online) The temperature dependence of the total energy of the graphene nanoflake $\mathrm{C}_{54}$ and the $\mathrm{H}$-passivated $\mathrm{C}_{54} \mathrm{H}_{20}$ using the REBO potential.

carbon structures. Zobelli et al. ${ }^{33}$ showed that DFTB theory accurately reproduced the structures and energies for a range of point defects such as vacancies and Stone-Wales defects in graphene. Migration barriers for vacancies and Stone-Walesdefect formation barriers are also accurately reproduced. Kuc et al. $^{27}$ studied the stability of graphene nanoflakes using DFTB theory and on comparing their results with those from DFT, good agreement was found between the two methods. Although this method is two orders of magnitude faster than DFT for the purpose of this work where we will study about 90 different configurations it will be computationally expensive. Therefore, we will use DFTB theory for a few $N$ values in order to show the accuracy of our classical MD simulation by considering one of the line carbons, two of the rings, five graphene nanoflakes, and six H-passivated systems.

\section{Lindemann criterion and specific heat}

The root-mean-square relative bond-length variance (Lindemann criterion) in addition to the caloric curve gives a reasonable computational method for determining the melting point of nanoclusters. It is sensitive to any change in the bond lengths at the microscopic scale. The Lindemann criterion ${ }^{34,35}$ is often used in molecular dynamics and Monte Carlo simulations in order to estimate the melting temperature in threedimensional bulk systems, ${ }^{36,37}$ two dimensional materials, ${ }^{20}$ and nanoclusters. ${ }^{38}$ We used the distance fluctuation of the Lindemann index $(\delta)$ in order to identify the melting temperature of our nanoclusters. For a system of $N$ atoms, the local Lindemann index for the $i$ th atom in the system is defined as ${ }^{39,40}$

$$
\delta_{i}=\frac{1}{N-1} \sum_{j(\neq i)} \frac{\sqrt{\left\langle r_{i j}^{2}\right\rangle_{T}-\left\langle r_{i j}\right\rangle_{T}^{2}}}{\left\langle r_{i j}\right\rangle_{T}}
$$

and the system-average Lindemann index is then given by

$$
\delta=\frac{1}{N} \sum_{i} \delta_{i},
$$

where $r_{i j}$ is the distance between the $i$ th and $j$ th atoms, $N$ is the number of atoms, and $\langle\cdots\rangle_{T}$ denotes the thermal average at temperature $T$. The Lindemann index ${ }^{41}$ depends on the specific system and its size, which varies in the range $0.03-0.15$, e.g., it was recently ${ }^{39}$ applied to nanoparticles and homopolymers and found to be in the range of $0.03-0.05$, for Ni nanoclusters it was found to be around $0.08,{ }^{38}$ and for carbon nanotubes about $0.03 .{ }^{21}$

For sufficiently low temperature there is no structural transition and the atoms exhibit thermal fluctuation around the $T=$ 0 equilibrium position. The oscillation amplitude increases linearly with temperaturein Hooke's regime for the atomic vibrations, leading to a linear increase of the Lindemann index with $T$. At higher temperature, anharmonic vibrations (nonlinear effects) become important and the Lindemann index exhibits a nonlinear dependence on $T$. The particle oscillation amplitude increases faster than linearly with $T$, but the system does not melt yet, since the arrangement of atoms has still some ordered structure, i.e., this is a solid-liquid coexistence state. For small nanoclusters the latter is related to ill-defined small three-dimensional structures. In general, melting occurs when the Lindemann index increases very sharply with $T$ over a small $T$ range. In this study we assume that the melting point is around the sharp jump in $\delta$, i.e., when the system becomes almost a random coil. We show that the Lindemann index adequately indicates the structural deformation (meltinglike transition) of carbon nanoclusters. The linear regime in $\delta$
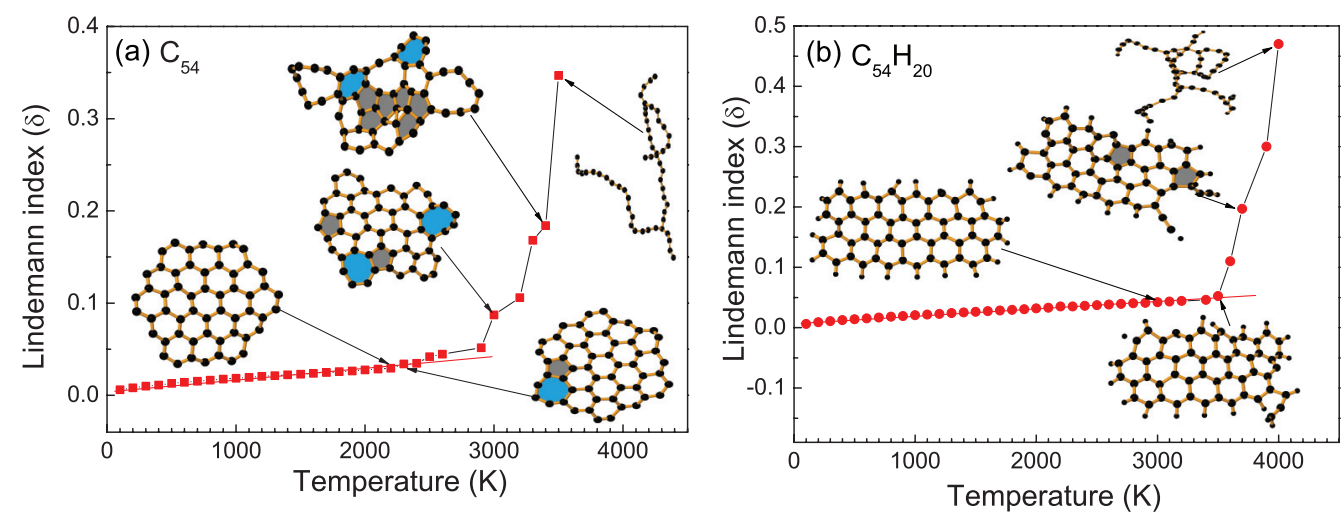

FIG. 3. (Color online) The temperature dependence of the Lindemann index for the cluster (a) without H-passivated $\mathrm{C}_{54}$ and (b) with $\mathrm{H}$-passivated $\mathrm{C}_{54} \mathrm{H}_{20}$. The insets show typical $\mathrm{C}$ (a) and $\mathrm{C}-\mathrm{H}$ (b) atom configurations, where the solid areas indicate topological defects. 

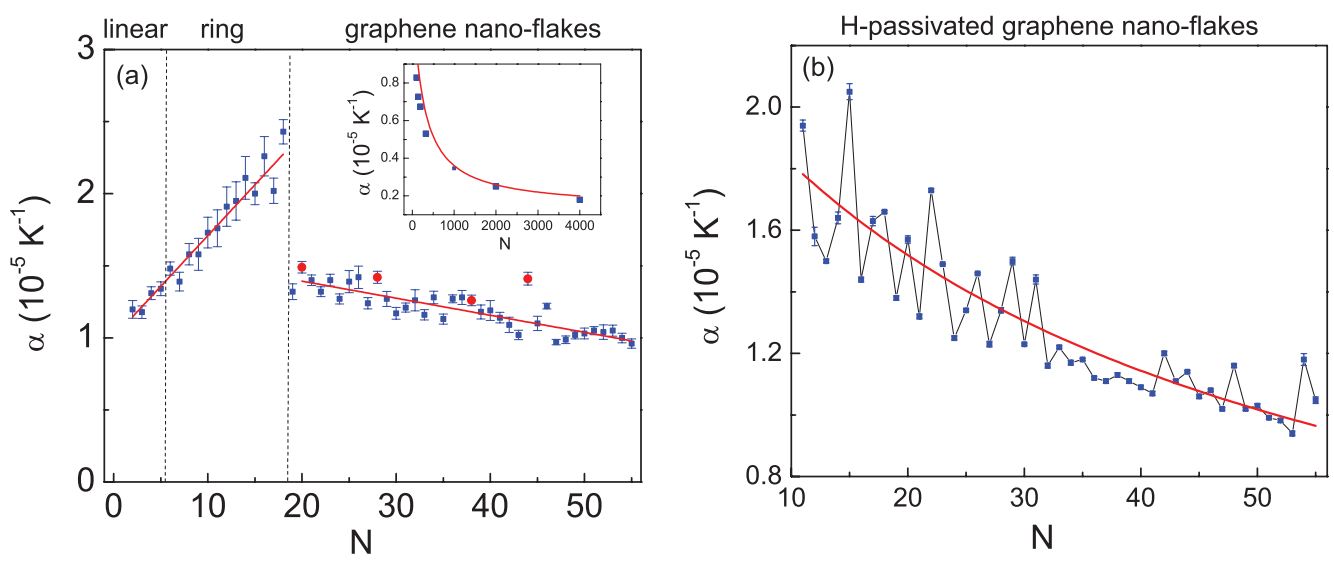

FIG. 4. (Color online) The low-temperature rate of change of the Lindmann index versus the number of atoms in (a) graphene nanoflakes and (b) H-passivated graphene nanoflakes. The red solid lines are linear fits to the average behavior of $\alpha$. The bowl-like clusters are shown by red solid circles (a). In the inset of (a), the same quantity is shown versus the number of atoms in graphene nanoflakes for $N$ up to 4000 .

obtained is smoother than in some of the previous studies ${ }^{38}$ for small nanoclusters. Therefore, we not only use the critical value of $\delta$ to determine the melting point but we also pay particular attention to the temperature dependence of the Lindemann index when identifying the melting temperature.

In addition to the Lindemann index, the total energy (caloric curve) and specific heat variation versus $T$ are two common quantities which can be used to determine the phase transition. We calculated the specific heat $C_{P}$ using the equation ${ }^{42}$

$$
C_{P}(T)=\frac{\left\langle E_{\text {total }}^{2}\right\rangle_{T}-\left\langle E_{\text {total }}\right\rangle_{T}^{2}}{k_{B} T^{2}},
$$

where $E_{\text {total }}=\sum_{i} \frac{1}{2} m_{i} v_{i}^{2}+E_{P}$. The average potential energy of the system was calculated as a function of temperature. In the crystalline state the total energy of the system increases almost linearly with temperature, and then after the critical temperature is reached, it increases more steeply, which is a signature of melting. We show that for a graphene nanoflake with 54 carbon atoms $\left(\mathrm{C}_{54}\right.$ and $\left.\mathrm{C}_{54} \mathrm{H}_{20}\right)$ the energy and heat capacity calculations are found to be consistent with the results for the melting temperature that we obtained from the analysis of the Lindemann index.

\section{RESULTS AND DISCUSSION}

\section{A. Energy}

The temperature dependence of the total energy of graphene nanoflakes $\left(\mathrm{C}_{54}\right.$ and $\left.\mathrm{C}_{54} \mathrm{H}_{20}\right)$ are depicted in Fig. 2 using the Brenner potential. For $\mathrm{C}_{54}$ the energy increases linearly at low temperatures and starts to deviate from the linear behavior around $T=2300 \mathrm{~K}$ due to the reconstruction of the zigzag edges and the formation of pentagon-heptagon (5-7) defects. This indicates that the first nucleation of melting starts around $2300 \mathrm{~K}$ and modifies the edges. As the temperature increases, the formation of pentagon, heptagon, 5-7, or 5-8-5 defects is possible and eventually large ring structures result in a dramatic increase of the energy. Above $T=3400 \mathrm{~K}$, there is a sharp increase in the energy showing a completely molten structure.

For $\mathrm{C}_{54} \mathrm{H}_{20}$, passivation removes the dangling orbitals of the $\mathrm{C}$ atoms at the edge, lowering the reactivity, and increasing the stability of the cluster. It was found that unlike the previous case (without $\mathrm{H}$-passivated clusters), the $\mathrm{H}$-passivated clusters keep their initial atomic arrangement up to higher temperatures and therefore no noticeable change in the geometry was found for temperatures up to $T=3200 \mathrm{~K}$ for $\mathrm{C}_{54} \mathrm{H}_{20}$. The binding energy of the H-passivated clusters is larger than for nonpassivated clusters. As the temperature increases further to $T=3500 \mathrm{~K}$, some hydrogen atoms start to dissociate, and finally the clusters convert into hydrocarbon chains around $T=4000 \mathrm{~K}$, showing a higher melting temperature than the corresponding nonpassivated cluster, which has a melting temperature of $3400 \mathrm{~K}$ (see Fig. 2).

\section{B. Lindemann index}

Figure 3 displays the variation of the Lindemann index with temperature for (a) $\mathrm{C}_{54}$ and (b) $\mathrm{C}_{54} \mathrm{H}_{20}$. The corresponding structures during heating for a few typical temperatures are shown in the insets. The slope of the function $\delta(T)$ (i.e., $\alpha=\frac{d \delta}{d T}$ ) is plotted in Fig. 4 for all studied structures of Fig. 1. The value of $\alpha$ (before reaching the melting point) increases monotonically for the carbon lines [Fig. 1(a)] and carbon rings [Fig. 1(b)]. This is an indication of keeping the

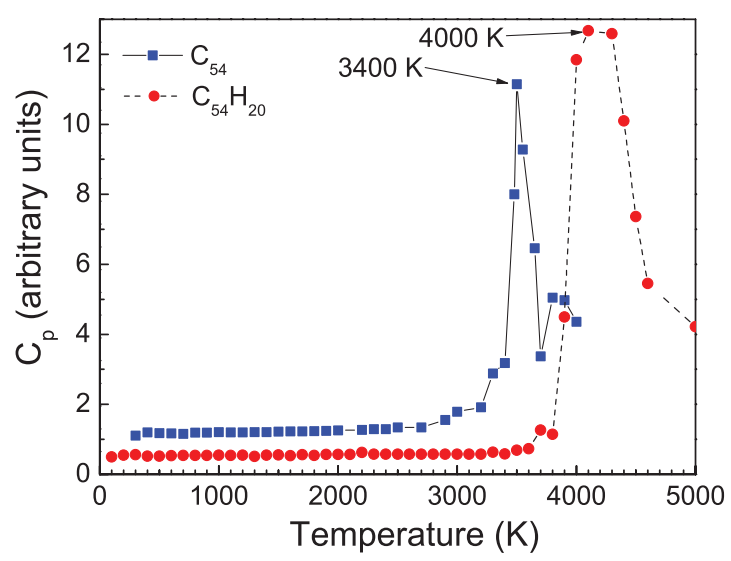

FIG. 5. (Color online) The temperature dependence of the specific heat for $\mathrm{C}_{54}$ and $\mathrm{C}_{54} \mathrm{H}_{20}$. 

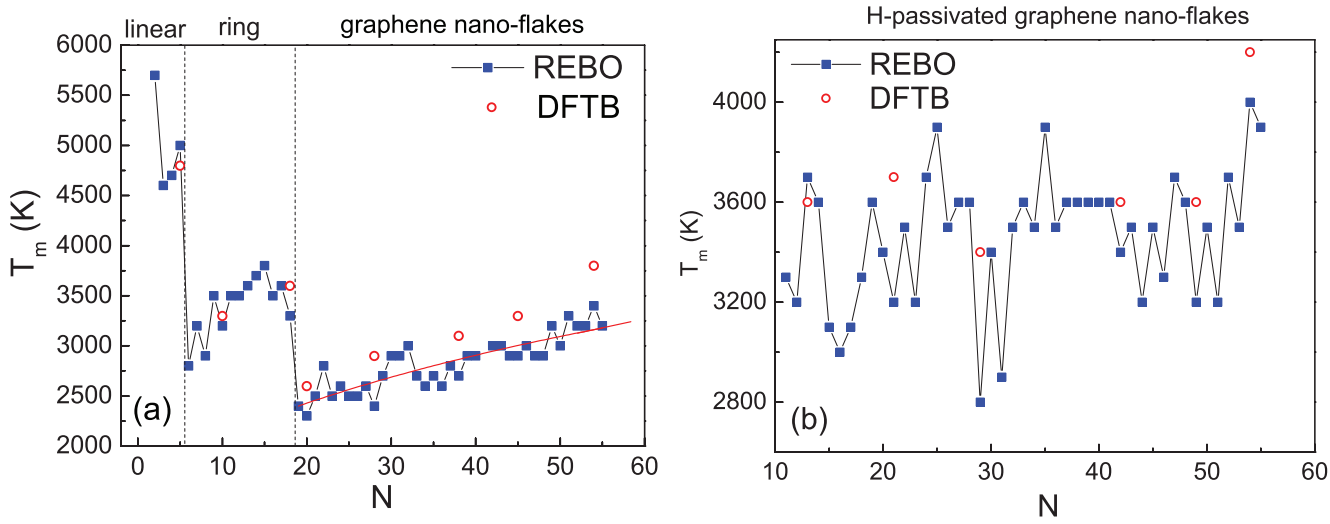

FIG. 6. (Color online) The melting temperature versus the number of atoms using the Brenner (square symbols) and DFTB (open circles) methods for (a) nanographene and (b) hydrogenated nanographene. The error bar is $50 \mathrm{~K}$.

initial configuration while nonlinear effects indicate defect formation, e.g., 5-7 defects in $\mathrm{C}_{54}$. The increase of $\alpha$ is fitted in Fig. 4 by the red line for $N \leqslant 18$ which is given by the function $\alpha(N)=a+b N$, where $a=(1.005 \pm 0.032) \times$ $10^{-5} \mathrm{~K}^{-1}$ and $b=(0.070 \pm 0.004) \times 10^{-5} \mathrm{~K}^{-1}$. Note that increasing temperature forces the system to be out of plane, e.g., the rings at finite temperature are not circles and become deformed ellipsoids in 3D.

For $N \geqslant 19$, there is a sudden decrease in $\alpha$ of size $\Delta \alpha=1.11 \times 10^{-5} \mathrm{~K}^{-1}$ due to the strong $s p^{2}$ bonds within the graphenelike clusters (instead of the simple covalent bonds in the carbon lines and rings) and a decreasing number of dangling bonds. The average behavior is fitted by the red line $\alpha(N)=a+b N$, where $a=(1.63 \pm 0.06) \times 10^{-5} \mathrm{~K}^{-1}$ and $b=(-0.012 \pm 0.001) \times 10^{-5} \mathrm{~K}^{-1}$. For bowl-like clusters ( $N=20,28,38,44)$, due to the presence of a topologically defective pentagon inside the cluster, the $\alpha$ value is larger as compared to their neighbor clusters. Therefore the important message is that dangling bonds and any kind of defect enhance anharmonic effects.

In order to investigate the effect of large sample sizes we also calculated $\alpha$ for a few large graphene nanoflakes and found that $\alpha$ decreases with $N$ (see the inset in Fig. 4). The maximum considered size of graphene nanoflakes had 4000 atoms. For large $N$, one expects saturation of $\alpha$; thus the line with negative slope which we fitted for $19 \leqslant N \leqslant 55$ should not be applicable. Therefore, we used the fit $\alpha(N)=$ $\alpha(\infty)+a /(1+b N)$, where $\alpha(\infty)=0.134 \times 10^{-5} \mathrm{~K}^{-1}, a=$ $(1.213 \pm 0.005) \times 10^{-5} \mathrm{~K}^{-1}$, and $b=(48.7 \pm 4.9) \times 10^{-4}$ on large clusters. These results clearly indicate that $\delta$ for small graphene nanoflakes is considerably larger than for larger flakes and graphene. Although the Lindemann index was defined initially in the thermodynamic limit (bulk material), we show that it is also a good parameter to investigate the effect of temperature and melting of nano-sized systems.

For completeness, we calculated $\alpha$ for H-passivated graphene nanoflakes for $11 \leqslant N \leqslant 55 \mathrm{C}$ atoms and found that $\alpha$, on average, decreases with $N$ [see Fig. 4(b)]. The average behavior is fitted by the red line $\alpha(N)=a /(1+b N)$, where $a=(2.262 \pm 0.115) \times 10^{-5} \mathrm{~K}^{-1}$ and $b=(0.024 \pm 0.003) \times$ $10^{-5}$. The rapid decrease in the latter fit [Fig. 4(b)] is an indication of the role of $\mathrm{H}$ passivation in making the graphene nanoflakes more stable against temperature for larger $N$.

\section{Specific heat}

The calculated specific heat curve for $\mathrm{C}_{54}$ is shown in Fig. 5 . A clear peak is observed in the specific heat with a maximum around $3400 \mathrm{~K}$, which we identify as the melting temperature, and which is close to the results from the analysis using the Lindemann index. The specific heat curve is also shown for $\mathrm{C}_{54} \mathrm{H}_{20}$ (see the red circles in Fig. 5) and displays a peak around $T=4000 \mathrm{~K}$, which is identified as the melting temperature, showing good agreement with the result of the previous caloric curve (see Fig. 2). A discontinuity or a sharp peak in the heat capacity is a clear indication of a phase transition. However, here this is not exactly a solid-to-liquid-like transition, but rather a nanoflake-to-random-coil transition.

\section{Melting temperature}

The melting temperature for all studied clusters $\mathrm{C}_{N}(2 \leqslant$ $N \leqslant 55)$ is shown in Fig. 6(a). The different parts separated by vertical dashed lines correspond to the three sets of systems shown in Figs. 1(a)-1(c). For small linear structures except for the carbon dimer the melting temperature increases with the number of atoms (linear chains have bond breaking).

In order to check if for large $N$ we approach the melting temperature of bulk graphene, we also calculated the melting temperatures of three large graphene nanoflakes and graphene (by performing a simulation having 4000 atoms with periodic boundary conditions in an NPT ensemble) and present the results in Table I. From Table I it is clear that large flakes slowly approach the melting temperature of graphene, i.e., $5500 \mathrm{~K}$. As a comparison the melting temperature reported in Ref. 20 by using the long-range carbon bond-order potential (LCBOP) potential was $4900 \mathrm{~K}$ and in Ref. 43 using the REBO potential was $5200 \mathrm{~K}$.

TABLE I. Melting temperature for large clusters.

\begin{tabular}{ll}
\hline \hline$N$ & $T_{m}(\mathrm{~K})$ \\
\hline 98 & 3800 \\
142 & 4000 \\
194 & 4100 \\
322 & 4200 \\
1000 & 4400 \\
Graphene & 5500 \\
\hline \hline
\end{tabular}


We fitted the melting temperature for graphenelike clusters [the red curve in Fig. 6(a)] by the function $T_{m}(N)=T_{m}^{b u l k}-$ $[a /(b+N)]$ where $a=(417 \pm 47) \times 10^{3} \mathrm{~K}, b=119.34 \pm$ 17.71 , and $T_{m}^{b u l k}=5500 \mathrm{~K}$ for graphene was taken from our simulation. We included the results of Table I in this fit. We also calculated the melting temperature using the DFTB method for the clusters with $N=5,10,18,20,28,38,45$, and $54\left(\mathrm{C}_{54}\right)$ which are represented by the open red circles in Fig. 6(a). They are found to slightly overestimate the

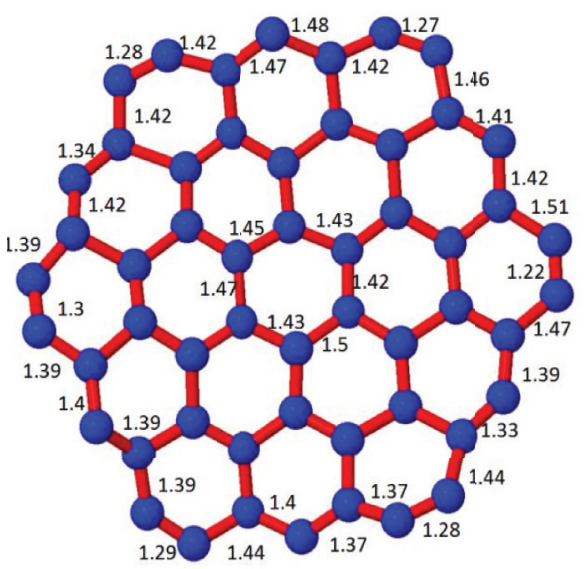

(a) $\mathrm{T}=1500 \mathrm{~K}$

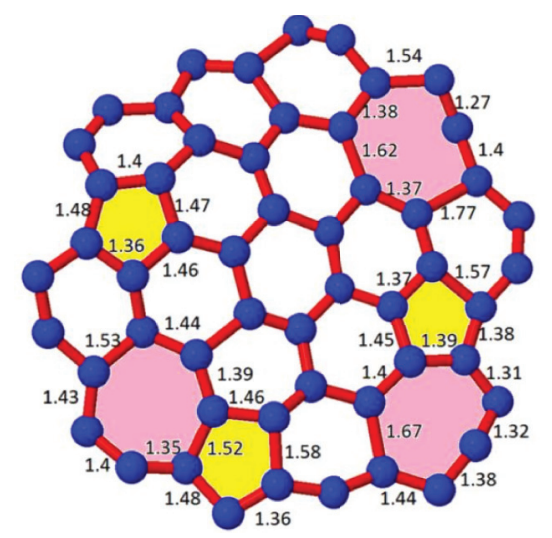

(c) $\mathrm{T}=2700 \mathrm{~K}$

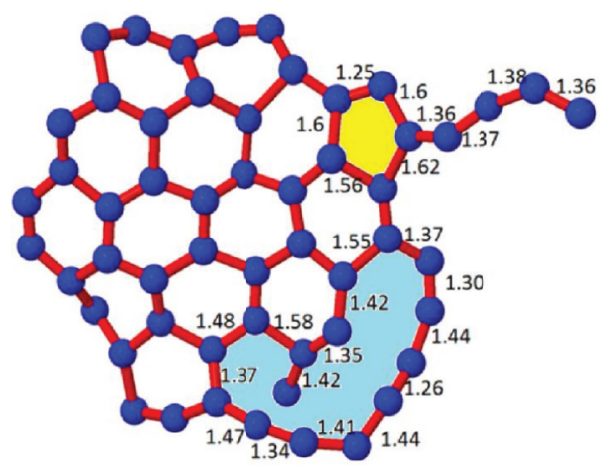

(e) $\mathrm{T}=3900 \mathrm{~K}$ melting temperature but exhibit clearly the same $N$-dependent trend.

For completeness, we also calculated the melting temperatures of $\mathrm{H}$-passivated clusters for $N=11-55 \mathrm{C}$ atoms [Fig. 6(b)]. The melting temperature fluctuates around $T=$ $3500 \mathrm{~K}$ (note that on average $T_{m}$ increases slowly with $N$ with large fluctuations imposed on it) for most of the clusters with minima for $N=29$ and $N=31 \mathrm{C}$ atoms due to the large number of defects in their structures. The clusters which have

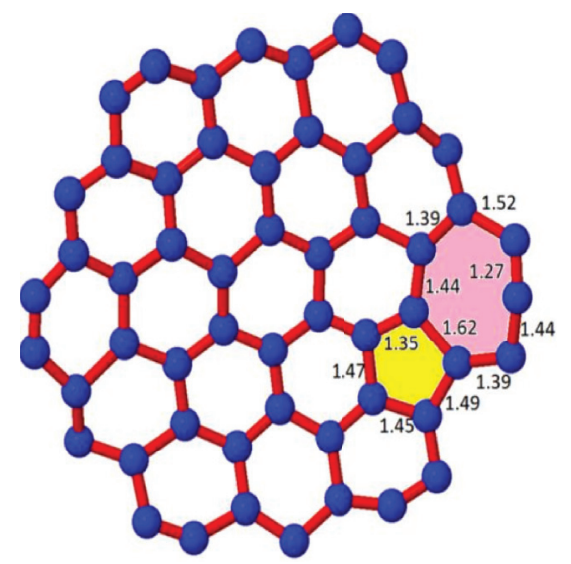

(b) $\mathrm{T}=2400 \mathrm{~K}$

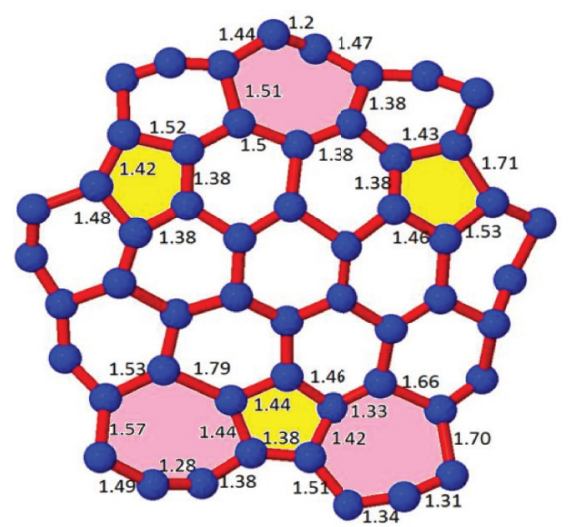

(d) $\mathrm{T}=3800 \mathrm{~K}$

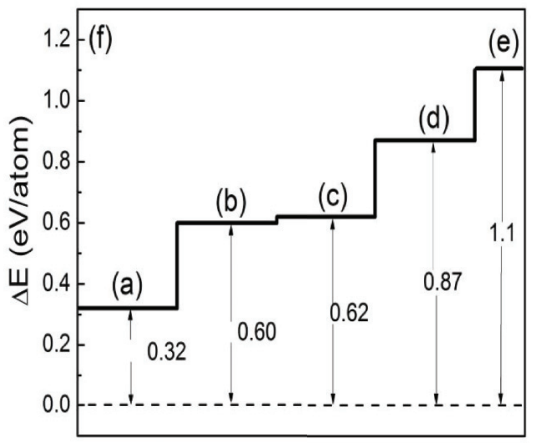

FIG. 7. (Color online) Five different snapshots of the cluster $\mathrm{C}_{54}$ at different temperatures (a)-(e). Bond lengths are indicated in the figure and the colored areas indicate defect structures. (f) The corresponding energy diagram for the five snapshots. 
pentagon defects on the boundary usually have lower melting temperature than the others. Here, the melting temperature for the clusters $N=13,21,29,42,49$, and 54 were also calculated using the DFTB method [open red circles in Fig. 6(b)]. In most cases the DFTB results are close to the Brenner potential results, indicating that the Brenner bond-order potential is a useful specialized potential for thermal effects in hydrocarbons.

\section{TOPOLOGY OF DEFECTS}

In this section we consider the topology of some of the defects which are created during the melting process of $\mathrm{C}_{54}$ using DFTB calculations. We found that these defects have a pronounced effect on the melting behavior of the system whose mechanism is different from those in both graphene and graphene nanoribbons. At low temperature the probability of defect creation is small and the flakes remain perfect, which for this low-temperature range is similar to the behavior of graphene ${ }^{20}$ and graphene nanoribbons. ${ }^{44}$ With increasing temperature (above about $2300 \mathrm{~K}$ ) the energy increases and the system can pass over certain potential barriers, and we found that during the molecular dynamics simulation the system transits from one metastable state to another; see Figs. 7(a) and 7(b). We show five snapshots of $\mathrm{C}_{54}$ at different temperature in Fig. 7. The transition temperature at which the edge reconstruction occurs, i.e., $T \sim 2400 \mathrm{~K}$, is lower than those found for defect formation in graphene, i.e., $3800 \mathrm{~K}$, and

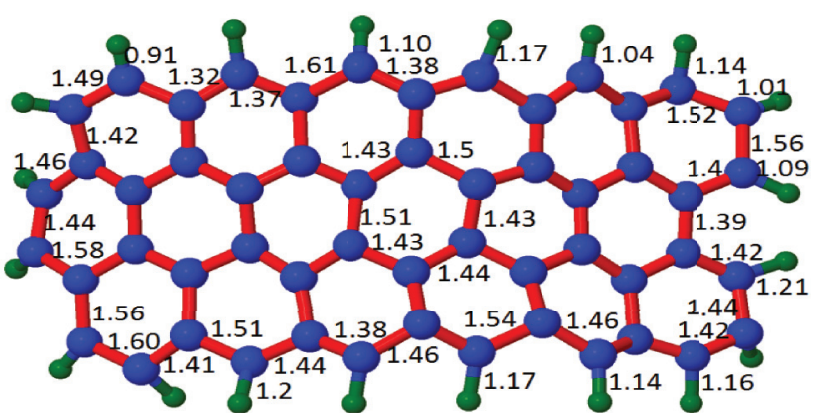

(a) $\mathrm{T}=3300 \mathrm{~K}$

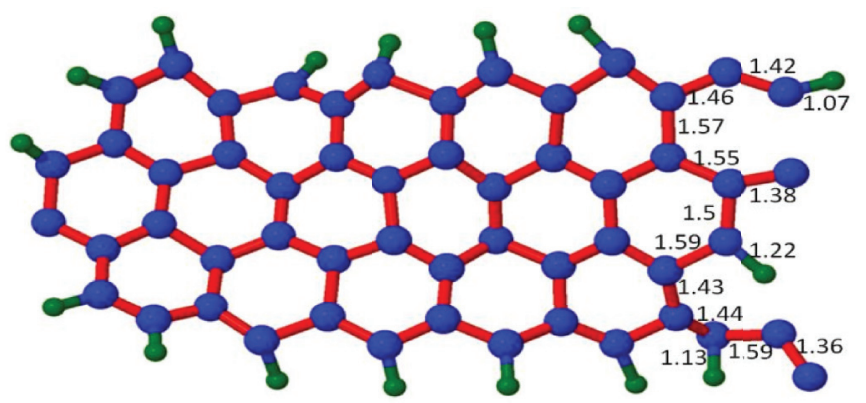

(c) $\mathrm{T}=4000 \mathrm{~K}$

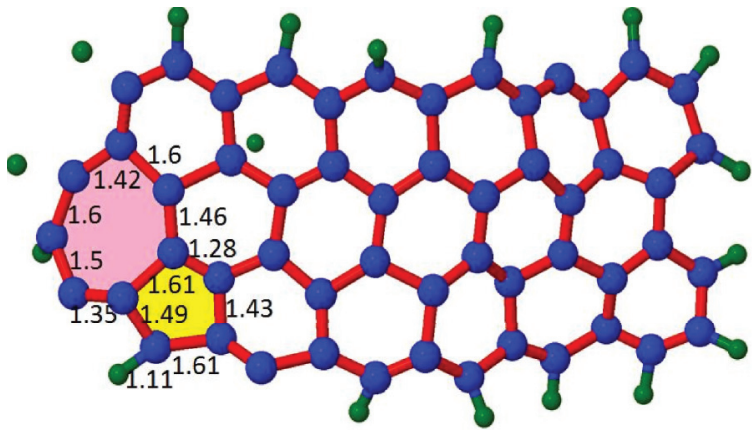

(b) $\mathrm{T}=3900 \mathrm{~K}$

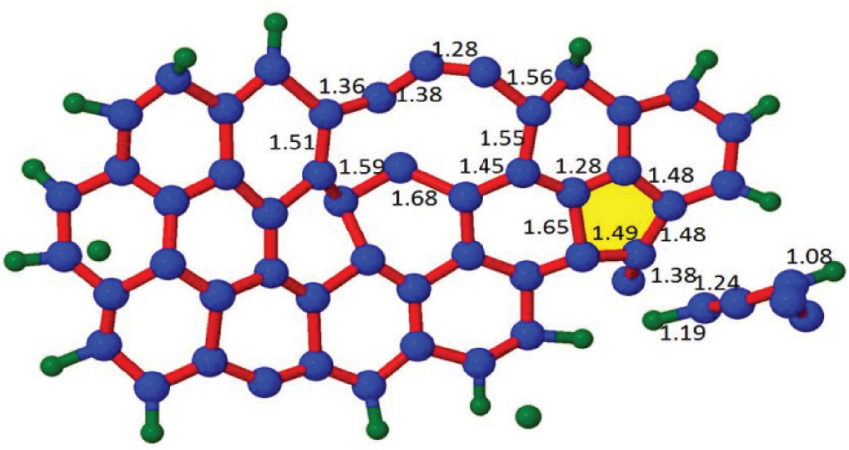

(d) $\mathrm{T}=4200 \mathrm{~K}$

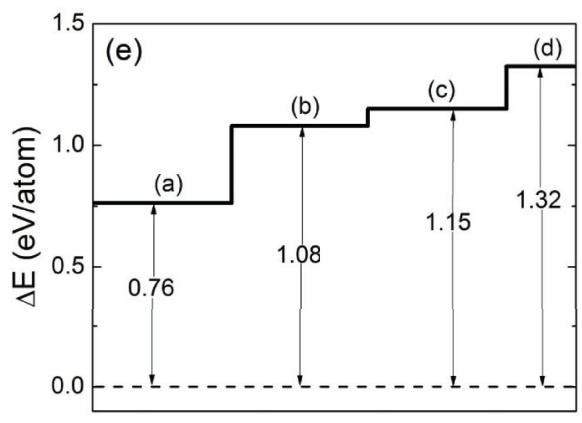

FIG. 8. (Color online) Four different snapshots of the cluster $\mathrm{C}_{54} \mathrm{H}_{20}$ at different temperatures (a)-(d). Bond lengths are indicated in the figure and the colored areas indicate defect structures. (e) The corresponding energy diagram for the four snapshots. 
edge reconstruction in graphene nano-ribbons, ${ }^{22}$ i.e., $2800 \mathrm{~K}$. As seen in Fig. 7(b) the first structure with defects has one heptagon and one pentagon at the edge with different bond lengths. The energy difference between $\mathrm{C}_{54}$ without [Fig. 7(a)] and with [Fig. 7(b)] defects is about $0.28 \mathrm{eV} /$ atom, which is due to the $900 \mathrm{~K}$ difference in the temperature between these two structures. In Fig. 7 the next configuration with defects due to a $1200 \mathrm{~K}$ increase in temperature is shown; it has more heptagon and pentagon defects (colored parts). By increasing the temperature further we found some transitions in the parts with defects, and even an edge reconstruction from heptagon to hexagon and vice versa, until the appearance of a tail-like part in the cluster [Fig. 7(e)]. The melting temperature for $\mathrm{C}_{54}$ as shown in Fig. 6(a) (open circles) is around $3900 \mathrm{~K}$. This melting temperature is lower than those found for graphene. The larger the graphene nanoflake, the higher the melting temperature. For small graphene nanoflakes the larger number of dangling bonds results in a lower melting temperature and larger boundary effects. The energy diagram for five snapshots is depicted in Fig. 7(f). The presented energy is the difference between the total energy of the system at a given temperature and the zero-temperature total energy for $\mathrm{C}_{54}$.

In Figs. 8(a)-8(d) we show the temperature effect on $\mathrm{C}_{54} \mathrm{H}_{20}$. In Fig. 8(e) the corresponding energy diagram is shown. Hydrogen atoms become released at $T=3900 \mathrm{~K}$. Notice that DFTB calculations for the configurations shown in Figs. 8(a)-8(d) were performed separately, i.e., we did not increase the temperature of the sample in Fig. 8(a) in order to obtain Fig. 8(b), but instead we performed four different calculations for these four snapshots. Thus we do not expect that we have sequential configurations.

\section{CONCLUSION}

Using molecular dynamics simulation and the Lindemann index for melting supplemented with results for the total energy and the specific heat, we investigated the melting of carbon nanoclusters. The melting temperature of small carbon flakes is lower than those for graphene and graphene nanoribbons. The Lindemann index is sensitive to temperature and is a good quantity for determining when structural deformations of the clusters start to occur. The melting temperature of small flakes on average increases versus the number of atoms in carbon nanoclusters. All clusters investigated show premelting behavior with different premelting intervals. For certain $N$ values defects are already present inside the cluster, which lowers the melting temperature. H-passivated clusters have a higher melting temperature than the non-H-passivated clusters with the same number of $\mathrm{C}$ atoms. The melting temperature for $\mathrm{H}$-passivated clusters is larger than for nonpassivated clusters. Our simulation results also help to understand the formation of defects (due to the increase of temperature) in the graphene nanoflakes, which can then be applied to understand the growth and thermal treatment of nanographene. We supplemented our analysis by DFTB calculations, which confirm the $N$ dependence of the melting temperature.

\section{ACKNOWLEDGMENTS}

This work was supported by the EU-Marie Curie IIF Postdoctoral Fellowship No. 299855 (for M.N.-A.), the ESFEuroGRAPHENE Project CONGRAN, the Flemish Science Foundation (FWO-Vl), and the Methusalem Foundation of the Flemish Government.
*SandeepKumar.Singh@ua.ac.be

†neekamal@srttu.edu

†Francois.Peeters@ua.ac.be

${ }^{1}$ J. G. Dash, Rev. Mod. Phys. 71, 1737 (1999).

${ }^{2}$ K. Koga, T. Ikeshoji, and K. I. Sugawara, Phys. Rev. Lett. 92, 115507 (2004).

${ }^{3}$ F. Ercolessi, W. Andreoni, and E. Tosatti, Phys. Rev. Lett. 66, 911 (1991).

${ }^{4}$ L. J. Lewis, P. Jensen, and J. L. Barrat, Phys. Rev. B 56, 2248 (1997).

${ }^{5}$ C. L. Cleveland, W. D. Luedtke, and U. Landman, Phys. Rev. B 60, 5065 (1999).

${ }^{6}$ A. Rytkönen, H. Häkkinen, and M. Manninen, Phys. Rev. Lett. 80, 3940 (1998).

${ }^{7}$ K. Joshi, D. G. Kanhere, and S. A. Blundell, Phys. Rev. B 66, 155329 (2002).

${ }^{8}$ F. C. Chuang, C. Z. Wang, S. Ögüt, J. R. Chelikowsky, and K. M. Ho, Phys. Rev. B 69, 165408 (2004).

${ }^{9}$ T. L. Beck and R. S. Berry, J. Chem. Phys. 88, 3910 (1988).

${ }^{10}$ B. S. Bas, M. J. Ford, and M. B. Cortie, J. Phys.: Condens. Matter 18, 55 (2006)

${ }^{11}$ M. Schmidt, R. Kusche, W. Kronmüller, B. von Issendorff, and H. Haberland, Phys. Rev. Lett. 79, 99 (1997).

${ }^{12}$ A. A. Shvartsburg and M. F. Jarrold, Phys. Rev. Lett. 85, 2530 (2000).
${ }^{13}$ G. A. Breaux, R. C. Benirschke, T. Sugai, B. S. Kinnear, and M. F. Jarrold, Phys. Rev. Lett. 91, 215508 (2003).

${ }^{14}$ C. Rey, L. J. Gallego, J. García-Rodeja, J. A. Alonso, and M. P. Iñiguez, Phys. Rev. B 48, 8253 (1993).

${ }^{15}$ J. García-Rodeja, C. Rey, L. J. Gallego, and J. A. Alonso, Phys. Rev. B 49, 8495 (1994).

${ }^{16}$ T. X. Li, Y. L. Ji, S. W. Yu, and G. H. Wang, Solid State Commun. 116, 547 (2000).

${ }^{17}$ A. H. Castro Neto, F. Guinea, N. M. R. Peres, K. S. Novoselov, and A. K. Geim, Rev. Mod. Phys. 81, 109 (2009).

${ }^{18}$ A. K. Geim and K. S. Novoselov, Nat. Mater. 6, 183 (2007).

${ }^{19}$ N. Alem, R. Erni, C. Kisielowski, M. D. Rossell, W. Gannett, and A. Zettl, Phys. Rev. B 80, 155425 (2009).

${ }^{20}$ K. V. Zakharchenko, A. Fasolino, J. H. Los, and M. I. Katsnelson, J. Phys.: Condens. Matter 23, 202202 (2011).

${ }^{21}$ K. Zhang, G. M. Stocks, and J. Zhong, Nanotechnology 18, 285703 (2007).

${ }^{22}$ G. D. Lee, C. Z. Wang, E. Yoon, N. M. Hwang, and K. M. Ho, Phys. Rev. B 81, 195419 (2010).

${ }^{23}$ D. P. Kosimov, A. A. Dzhurakhalov, and F. M. Peeters, Phys. Rev. B 78, 235433 (2008).

${ }^{24}$ K. Raghavachari and J. S. Binkley, J. Chem. Phys. 87, 2191 (1987).

${ }^{25}$ D. P. Kosimov, A. A. Dzhurakhalov, and F. M. Peeters, Phys. Rev. B 81, 195414 (2010). 
${ }^{26}$ D. W. Brenner, O. A. Shenderova, J. A. Harrison, S. J. Stuart, B. Ni, and S. B. Sinnott, J. Phys.: Condens. Matter 14, 783 (2002).

${ }^{27}$ A. Kuc, T. Heine, and G. Seifert, Phys. Rev. B 81, 085430 (2010).

${ }^{28}$ L. Rui, H. Yuan-Zhong, W. Hui, and Z. Yu-Jun, Chin. Phys. 17, 4253 (2008).

${ }^{29}$ D. Porezag, T. Frauenheim, T. Kohler, G. Seifert, and R. Kaschner, Phys. Rev. B 51, 12947 (1995).

${ }^{30}$ M. Elstner, D. Porezag, G. Jungnickel, J. Elsner, M. Haugk, T. Frauenheim, S. Suhai, and G. Seifert, Phys. Rev. B 58, 7260 (1998).

${ }^{31}$ T. Frauenheim, G. Seifert, M. Elstner, T. Niehaus, C. Kohler, M. Sternberg, Z. Hajnal, A. Di Carlo, and S. Suhai, J. Phys.: Condens. Matter 14, 3015 (2002).

${ }^{32}$ G. Zheng, S. Irle, and K. Morokuma, Chem. Phys. Lett. 412, 210 (2005).

${ }^{33}$ A. Zobelli, V. Ivanovskaya, P. Wagner, I. Suarez-Martinez, A. Yaya, and C. P. Ewels, Phys. Status Solidi B 249, 276 (2012).

${ }^{34}$ N. H. March and M. P. Tosi, Introduction to Liquid State Physics (World Scientific, Singapore, 2002).
${ }^{35}$ J. M. Ziman, Principles of the Theory of Solids (Cambridge University, Cambridge, 1972).

${ }^{36}$ K. Sokolowski-Tinten, C. Blome, J. Blums, A. Cavalleri, C. Dietrich, A. Tarasevitch, I. Uschmann, E. Förster, M. Kammler, M. Horn-von-Hoegen, and D. von der Linde, Nature (London) 422, 287 (2003).

${ }^{37}$ G. Shen, V. B. Prakapenka, M. L. Rivers, and S. R. Sutton, Phys. Rev. Lett. 92, 185701 (2004).

${ }^{38}$ E. C. Neyts and A. Bogaerts, J. Phys. Chem. C 113, 2771 (2009).

${ }^{39}$ Y. Zhou, M. Karplus, K. D. Ball, and R. S. Berry, J. Chem. Phys. 116, 2323 (2002).

${ }^{40}$ F. Ding, K. Bolton, and A. Rosen, Eur. Phys. J. D 34, 275 (2005).

${ }^{41}$ F. A. Lindemann, Phys. Z. 11, 609 (1910); H. Lowen, Phys. Rep. 237, 249 (1994); J. H. Bilgram, ibid. 153, 1 (1987).

${ }^{42}$ P. J. Hsu, J. S. Luo, S. K. Lai, J. F. Wax, and J.-L. Bretonnet, J. Chem. Phys. 129, 194302 (2008).

${ }^{43}$ B. Steele, R. Perriot, V. Zhakhovsky, and I. Oleynik, http://meetings.aps.org/Meeting/MAR12/Event/166282.

${ }^{44}$ P. Koskinen, S. Malola, and H. Häkkinen, Phys. Rev. Lett. 101, 115502 (2008). 